SCHNEIDER, K. (1992) Klinische Psychopathologie (14th edn). Stuttgart: GeorgThieme Verlag.

SLATER, E. \& ROTH, M. (1979) Clinical Psychiatry (3rd edn). London: BaillièreTindal.

Claus Langmaack Specialist Registrar in Genera Adult Psychiatry, South Devon Drug and Alcohol Service, 8 Morgan Avenue, TorquayTQ2 5RS; e-mail: langmaack $@$ doctors.org.uk

\section{Enough of the sticks, what about some carrots?}

Sir: Palmer \& Lelliott describe some important aspects of guideline implementation (Psychiatric Bulletin, March 2000, 24, 90-93). Encouraging doctors to change their practice is a difficult task. Examples of published audits confirm this (Duffet \& Lelliott, 1998).

As well as considering vigorous strategies for implementing change, we wonder if more emphasis should be placed on provision of reward systems for guideline compliance. Doctors prefer to rely on clinical experience for their decisionmaking (Dickson-Mulinga, 1998). In contrast, guidelines can be viewed as sinister threats to this professiona autonomy. Clinical experience follows an operant learning model. 'Good' clinical decisions are rewarded by patient improvement. 'Poor' clinical decisions are so labelled because they result in patient deterioration. The perceived reward for following guidelines must be greater than the integral reward predictable from a good 'clinical experience'-based decision.

General practitioners receive financial incentives for reaching targets for preventative medicine interventions. If a change in practice is perceived as an increased workload, financial compensation can soften the blow. Rewarding high quality practice makes sense. Although this may add to the unit price of change, this system may prove cost effective. Further research evaluation may be indicated, of course. Producing and disseminating guidelines that nobody reads or follows, is surely an ultimate waste of time and money.

\section{References}

DICKSON-MULINGA, J. (1998) Rationale behind psychiatrists' choice of drug. Psychiatric Bulletin, 22 677-680.

DUFFET, R. \& LELLIOTT, P. (1998) Auditing electroconvulsive therapy. The third cycle. British Journal of Psychiatry, 172, 401-405.
*Sian Llewellyn-Jones Senior House Officer in Psychiatry, Sally Cubbin Senior House Officer in Psychiatry, Swansea National Health Service Trust, Cefn Coed Hospital, Waunarlwydd Road, Swansea SA2 OGH

\section{Substance use and misuse in psychiatric wards}

Sir: I was interested to read the overview provided by Williams \& Cohen (Psychiatric Bulletin, February 2000, 24, 43-46).

In our hospital, we have found that establishing a close liaison with our local police service to advise us and support us has been helpful in dealing with this issue. I am aware that some units have used 'sniffer dogs' on wards to give the message that illicit drugs are illegal, and having an occasional police presence does at least deter dealers from visiting the wards.

The local police have also been helpful in providing drug awareness sessions for our staff and offering advice and support in dealing with difficult problems, taking a non-judgmental approach.

G. L. Milner Consultant Psychiatrist, Solihul Healthcare NHS Trust Mental Health Services, First Floor, 51 Grove Road, Solihull, West Midlands B912AQ

\section{Royal College Music Society}

Sir: Although I am not a golfer I was delighted to read Chris Thompson's account of the inaugural meeting of the Royal College Golf Society and would wish to give my congratulations to Robert Jackson. I note that this Society is planning a further meeting at the end of the Annual General Meeting in Edinburgh.

My prowess in golf is more limited than my interest in music. Most of our now defunct mental hospitals used to have sizeable orchestras, some of them conducted by their medical directors. Edward Elgar used to conduct the Powyck Mental Hospital Choir and composed music specifically for its musicians. There is now a broader consensus that psychiatry is a science, as well as an art, certainly that psychiatrists and other mental health professionals have both left and right hemispheres which need exercising. Judging by the curriculum vitaes of many of my colleagues and of trainees applying for postgraduate posts, there is an abundance of artistic talent among our membership. I will try to convene an inaugural meeting of anyone interested in pursuing these specific matters further. If there is sufficient interest we could even arrange a very fringe event in Edinburgh during, or shortly after, the formal proceedings.

John L. Cox President, Royal College of Psychiatrists, 17 Belgrave Square, London SW1X 8PG

\section{2nd PAN conference in Kathmandu}

Sir: The Second International Conference of the Psychiatrists' Association of Nepal (PAN) was held on 13-14 November 1999 in Kathmandu. The lecture entitled 'Mental Health at the Doorsteps' marked the PAN journal launch. The conference centre was a large hotel where hospitality was generous, and the gardens provided a relaxed setting for networking.

Delegates from Asia, Australia and Britain attended. The topics included: mental health in women; psychiatric training of health workers and its evaluation; liaison with traditional healers; child psychiatry in the context of a changing culture; substance misuse, and mental health needs of a growing elderly population.

Many speakers referred to two major constraints on mental health care delivery: the very small number of psychiatrists (20 for a population of 21 million) and the provision in remote areas The introduction of telepsychiatry to overcome these constraints was canvassed and debated. The expenditure required for the network could be offset by the consultation costs for patients and their relatives who customarily accompany a patient (cost of journey, food, accommodation, loss of income during travel and the hospital attendance - it is common to travel for several days for an appointment). Reduced patient-doctor contact was a concern regarding telepsychiatry, however, it should not be seen as a substitute for psychiatric consultation and teaching, but as a pragmatic alternative to the absence of service provision in remote areas.

The Kathmandu Valley provided an unforgettable setting. This will be an annual event and information can be obtained from the Mental Health Resource Centre, e-mail: sregmi@healthnet.org.np.

Eleanor Mullen Visiting Lecturer and Consultant in Old Age Psychiatry, Department of Psychiatry and Mental Health, Tribhuvan UniversityTeaching Hospital, PO Box 2434 Kathmandu, Nepal, Helena Novek Drug and Alcohol Department, Royal Prince Alfred Hospital, Missenden Road, Camperdown, NSW 2050, Australia 